\title{
Insulin pumps in general practice
}

\author{
Barbora Paldus \\ Endocrinologist ${ }^{1}$ \\ Research fellow ${ }^{2}$ \\ Melissa H Lee \\ Endocrinologist', 13 \\ Research fellow ${ }^{2}$

\section{David N O'Neal} \\ Professor ${ }^{2}$ \\ Endocrinologist $t^{1,3}$ \\ ' Werribee Mercy Hospital, \\ Werribee, Victoria \\ ${ }^{2}$ Department of Medicine, \\ University of Melbourne \\ ${ }^{3}$ St Vincent's Hospital, \\ Melbourne
}

\section{Keywords}

diabetes, glucose, insulin, insulin infusion systems, ketoacidosis

Aust Prescr 2018:41:186-90

https://doi.org/10.18773/ austprescr.2018.056

\section{SUMMARY}

Insulin pumps deliver continuous subcutaneous rapid-acting insulin in a flexible manner. In Australia their main use is in the management of type 1 diabetes.

The care of patients with type 1 diabetes on an insulin pump should involve a team approach which includes the GP as well as specialists in diabetes. The GP should therefore understand the terminology associated with insulin pump therapy. Technical support is available from the manufacturer's helpline.

If blood glucose is significantly elevated or the patient is nauseated, blood ketones should be checked. When ketones are elevated the insulin delivery line should be changed, and referral to an emergency department may be needed.

\section{Introduction}

People with type 1 diabetes have an absolute deficiency in islet cell function which requires therapy with injections of insulin. Despite advances in injection equipment and modern insulin formulations with favourable pharmacokinetic profiles, there remain limitations in the flexibility and responsiveness of injected insulin. For example, there is very little opportunity to influence basal insulin delivery after an injection of long-acting insulin. Some patients (for example those who have completed courses such as Dose Adjustment for Normal Eating) can make appropriate adjustments to their dose of rapid-acting insulin. However, adjustments are usually constrained by the increments in the dose of insulin that are able to be administered and the patient's ability to perform calculations to account for meals and to correct for glucose concentrations outside a healthy range. These constraints are particularly apparent in people with type 1 diabetes as insulin requirements can vary significantly and rapidly. Insulin pumps have been developed to address these shortcomings.

In Australia insulin pumps are almost entirely used for the management of people with type 1 diabetes. In the USA, in addition to those with type 1 diabetes, there are a significant number of people with type 2 diabetes who use insulin pumps.

\section{Insulin pumps}

Since the commercialisation of the first subcutaneous insulin pumps in the 1970s, pump therapy has changed dramatically. It has evolved from large bulky devices that could only deliver a single basal rate and required patients to calculate bolus doses, to today's pager-sized devices with increasingly sophisticated technology. Insulin pump therapy is rapidly becoming the standard of care for the management of type 1 diabetes.

Insulin pumps vary in size and appearance depending on the model, but the basic set-up is common to most models. The pump houses an insulin cartridge or 'reservoir'. This reservoir is connected to an insulin delivery line which in turn is connected to a subcutaneous cannula that delivers insulin to the patient (Fig. 1). The reservoir, delivery line and cannula generally need to be replaced every three days. Just as with injected insulin, the patient should rotate the site for the insertion of the canula.

\section{Dosing}

When commencing insulin pump therapy the amount of rapid-acting insulin to be delivered by the pump is estimated from calculations based on the total daily dose of injected insulin. Usually a $20 \%$ reduction in the total daily dose of insulin is estimated as the appropriate total daily dose for pump therapy. Half of this estimate is delivered by the pump to meet basal insulin needs. The initial bolus doses of insulin for meals are estimated using the 'rule of 500' where the number 500 is divided by the total daily injected dose of insulin in units to give an insulin-tocarbohydrate ratio.

The four main settings that need to be programmed into an insulin pump are:

- basal dosing (basal rates)

- bolus dosing (insulin-to-carbohydrate ratio)

- blood glucose correction settings (insulin sensitivity factor also known as the correction factor)

- insulin action time. 
The pump only holds and delivers rapid-acting insulin, so basal dosing is given as an hourly rate. For example, 24 units daily of a long-acting insulin would be equivalent to one unit per hour of rapid-acting insulin as a continuous infusion. Basal dosing via an insulin pump is much more flexible than long-acting insulin injections because different basal rates can be given at different times of the day. Most pump wearers have between one and six different basal rates over 24 hours.

Current generation insulin pumps do not automatically administer insulin boluses. Bolus administration is initiated by the patient. The bolus doses of rapidacting insulin delivered by a pump's bolus calculator may have two components. The first represents an amount of insulin calculated to address the glucose influx associated with a meal. This dose is calculated with the pump's insulin-to-carbohydrate ratio.

For meals, the pump delivers one unit of insulin for every specified amount of carbohydrates. For example, for a meal containing $20 \mathrm{~g}$ carbohydrate with an insulin-to-carbohydrate ratio of 1 unit of insulin/10 g carbohydrate, the pump would deliver 2 units of insulin. When adjusting the insulin-to-carbohydrate ratio it should be noted that a decrease in the number of grams of carbohydrate per unit of insulin results in an increase in insulin dose. For example, a change from 1 unit of insulin/15 g carbohydrate to 1 unit of insulin/10 g carbohydrate increases the amount of insulin delivered by one third.

When the person's blood glucose is in the target range no additional insulin is administered with the bolus. If the glucose is elevated, the pump's bolus calculator may administer a second component to the bolus to bring the concentration down into the target range. This additional dose of insulin is based on the insulin sensitivity factor. If the person is not eating and the glucose concentration is above the target range, a bolus of rapid-acting insulin may be delivered purely to correct the elevated glucose.

Bolus doses of insulin for corrections are calculated automatically by the insulin pump using the patient's finger-prick glucose (entered via a compatible blood glucose meter) and the pre-programmed insulin sensitivity factor. This factor is the amount that blood glucose $(\mathrm{mmol} / \mathrm{L})$ is lowered by one unit of insulin. For example, for an entered blood glucose of $7 \mathrm{mmol} / \mathrm{L}$ and a pre-set target blood glucose of $5 \mathrm{mmol} / \mathrm{L}$, an insulin sensitivity factor of $2 \mathrm{mmol} /$ unit would result in the delivery of 1 unit of insulin.

Insulin action time refers to an estimate of the duration of the activity of the insulin that has already been delivered by the pump subcutaneously. It provides an estimate of the residual activity of rapidacting insulin delivered by a previous bolus thereby

\section{Fig. 1 Components of an insulin pump}

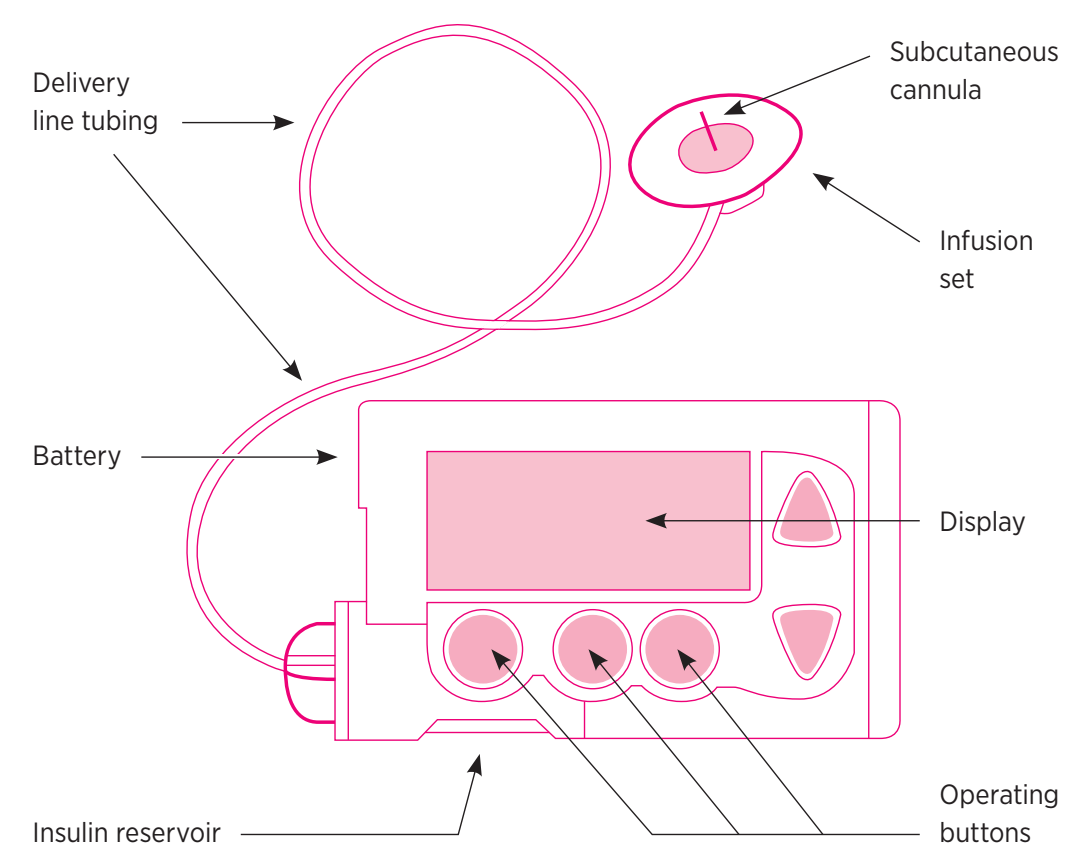

modulating the subsequent delivery of further boluses of insulin. This modulation is aimed at reducing the risk of hypoglycaemia by avoiding the 'stacking' of insulin with multiple boluses.

\section{Monitoring}

Continuous glucose monitors are an increasingly popular adjunct to insulin pump therapy (Fig. 2). They allow for real-time monitoring of glucose concentrations via a small subcutaneous electrode or sensor. The sensor measures glucose in the subcutaneous interstitial fluid and sends this information directly to the pump (or a handheld receiver) every five minutes. More recent advances in technology have allowed for insulin pumps to automatically shut off insulin delivery for two hours in the event of hypoglycaemia ('low-glucose suspend') or predicted hypoglycaemia ('predictive low-glucose suspend').

\section{Benefits of pump therapy}

Unlike insulin pens or injections, data can be uploaded from most insulin pumps via web-based software. The data relating to glucose concentrations and insulin delivery can be reviewed by the health professional in conjunction with the patient. The GP should instruct the patient to download PDFs of these reports and bring them to their appointment.

Meta-analyses and randomised controlled trials have reported improvements in glycaemic control using insulin pump therapy compared to multiple 


\section{Fig. 2 Continuous glucose monitoring}

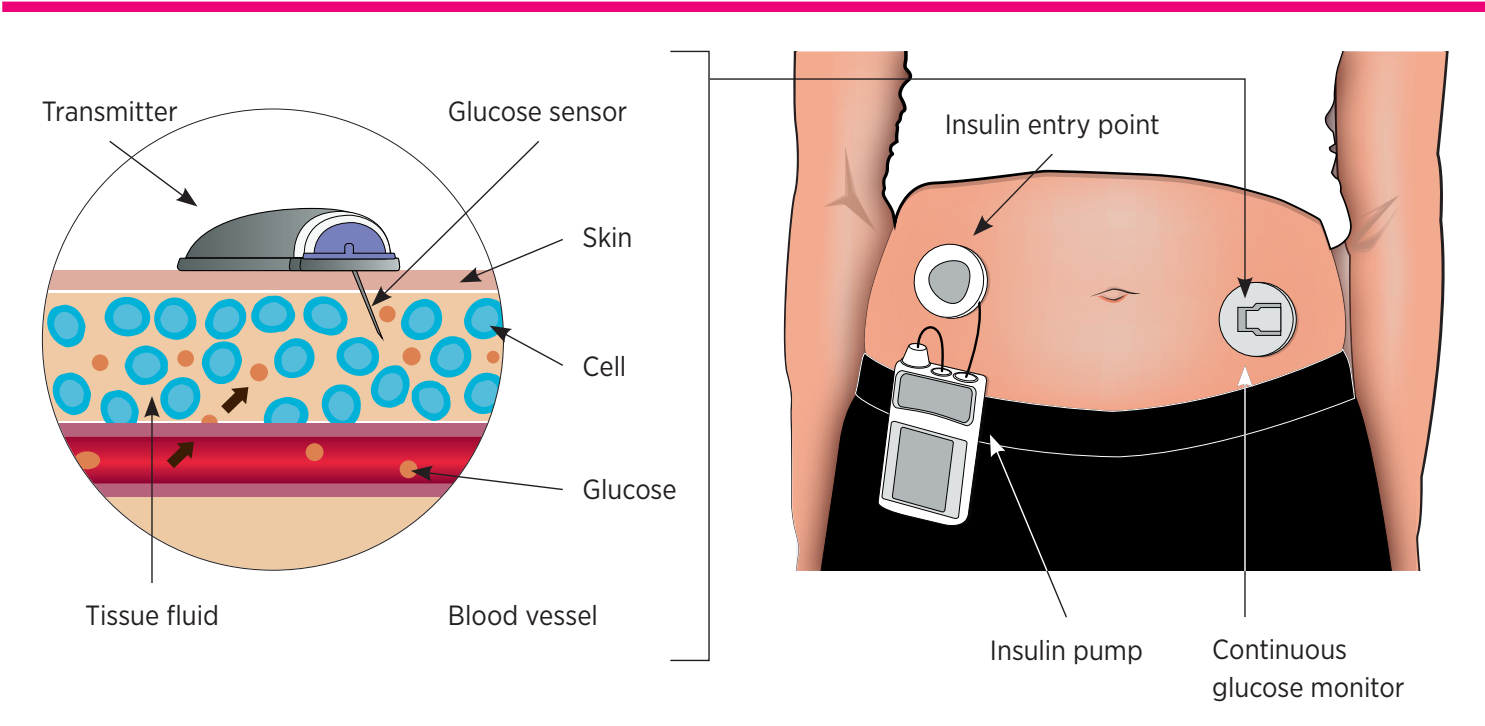

Reproduced with permission from https://www.ontrackdiabetes.com/type-1-diabetes/what-continuous-glucose-monitor-cgm

daily injections. 'These include reductions in blood glucose, reduced hypoglycaemia (both frequency and severity), lower glycated haemoglobin (HbAlc), lower insulin requirements, and improved quality of life. The greatest motivating factor for Australians with type 1 diabetes to use an insulin pump is to improve their diabetes control. ${ }^{2}$

There are numerous benefits in using insulin pump therapy rather than multiple daily injections. However, it is important to recognise that there may also be some disadvantages (Box).

\section{Box Advantages and disadvantages of insulin pumps}

\section{Advantages \\ Provides insulin delivery that better mimics normal pancreatic function \\ Uses rapid-acting insulin only and minimises glucose peaks and absorption-related variability}

Built-in bolus algorithms to suggest bolus doses based on user-estimated carbohydrate content and blood glucose

Increased flexibility with day-to-day activities e.g. exercise, mealtimes, travel, shift work Can be integrated with continuous glucose monitoring to provide real-time glucose information

Potential for advanced features e.g. low-glucose suspend, predictive low-glucose suspend

\section{Disadvantages}

External device attached to the body 24 hours a day

Potential for mechanical malfunction such as blockage or kinking in the infusion set which can lead to diabetic ketoacidosis

Potential for insertion site problems such as skin infections or local irritation

High cost and limited access for uninsured patients

Patients have to be willing and able to manage the technology

\section{Suitability for pump therapy}

Careful consideration is essential to determine which patients are suitable for insulin pump therapy. Patients need to be willing and able to self-manage the technology which requires programming by the user. The patient also needs adequate carbohydrate counting skills, and to be competent at blood glucose self-monitoring. They must be motivated to accept responsibility for their own care.

Insulin pumps are typically recommended for patients with suboptimal glycaemic control despite multiple daily injections. In particular, those with frequent or unpredictable hypoglycaemia associated with hypoglycaemia unawareness may benefit. Women planning pregnancy often transition to pump therapy before conception to achieve tighter glycaemic targets pre-pregnancy and to maintain control during pregnancy. Insulin pumps may also provide greater flexibility for people who do shift work, frequent travel, intensive exercise and physical activity.

\section{Access to insulin pumps}

In Australia, insulin pump therapy is limited to people with type 1 diabetes who have private health insurance. There are some government subsidy programs and charitable organisations, such as the Juvenile Diabetes Research Foundation (JDRF), that may assist with the cost of an insulin pump in special circumstances. Patients without private health insurance have to purchase the insulin pump themselves. The current commercial price of an insulin pump is approximately $\$ 10000$. 
In 2017 a government subsidy for continuous glucose monitors was implemented for people under the age of 21 years. However, patients over 21 years receive no subsidy and must purchase continuous glucose monitors at the full price of approximately \$300-400 per month. Additional costs with pump use include consumables such as infusion sets and reservoirs, costing $\$ 25-30$ per month.

\section{Managing insulin pumps in general practice}

The management of a patient on an insulin pump requires the involvement of a specialist diabetes team including an endocrinologist, diabetes nurse educator and dietitian. The GP is an essential member of this team and it is therefore important GPs understand the terminology related to insulin pump therapy (Table). ${ }^{3}$

As a rule, the GP is not routinely involved in altering the pump settings but changes to insulin delivery may be performed in partnership with the specialist team. The GP is not expected to know all things about all pumps and the patient should be able to guide the doctor through the 'button pushing'. In general, the basal insulin delivery determines overnight and pre-meal glucose concentrations, while bolus insulin determines post-prandial glucose levels. Bolus insulin is also used to correct high glucose levels.

It is essential that the patient has prescriptions for rapid-acting insulin for their pump. In addition, they require pens and needles as a backup for injections of rapid- and long-acting insulin, should the pump fail or should they need to temporarily discontinue insulin pump therapy (e.g. while white water rafting). While the pump is disconnected and suspended during swimming and bathing, alternate means of insulin delivery are usually not required as these activities are usually of limited duration. Nevertheless, it is important that the patient reconnect the pump and cancel the suspension of insulin delivery after their activity. This is important because insulin pumps only deliver rapid-acting insulin. After 4-6 hours of a pump being disconnected insulin activity will fall to zero with a risk of ketoacidosis. If the pump is not able to be reconnected, insulin will need to be given by injection. The patient should also have a current glucagon injecting kit and the relevant people, such as family members, should be educated in its use. Details of the patient's insulin pump settings at the time of their most recent consultation should be documented in the patient's general practice record. These details include:

- insulin-to-carbohydrate ratios (which may differ through the day)

- basal insulin delivery settings

- insulin action time

- insulin sensitivity factor

- total daily insulin and the amount given as basal insulin.

\section{Table Insulin pump terminology ${ }^{3}$}

\begin{tabular}{|c|c|c|}
\hline Terminology & Definition & Example \\
\hline Basal rate & $\begin{array}{l}\text { Background delivery of small doses of insulin at } \\
\text { programmed rates (measured in units per hour) }\end{array}$ & $1 \mathrm{U} / \mathrm{hr}$ delivers 1 unit of insulin every hour \\
\hline Bolus dose & $\begin{array}{l}\text { Discrete and more rapid delivery of insulin for food or blood } \\
\text { glucose corrections (measured in units) }\end{array}$ & 5 unit bolus delivers 5 units of insulin quickly \\
\hline $\begin{array}{l}\text { Insulin-to-carbohydrate } \\
\text { ratio }\end{array}$ & $\begin{array}{l}\text { Number of grams of carbohydrate that one unit of insulin } \\
\text { will cover (measured in grams per unit) }\end{array}$ & $10 \mathrm{~g} /$ unit delivers 1 unit for every $10 \mathrm{~g}$ carbohydrate \\
\hline $\begin{array}{l}\text { Insulin sensitivity } \\
\text { (correction) factor }\end{array}$ & $\begin{array}{l}\text { Amount of blood glucose ( } \mathrm{mmol} / \mathrm{L} \text { ) that is lowered by one } \\
\text { unit of insulin (measured in } \mathrm{mmol} / \mathrm{L} \text { per unit) }\end{array}$ & $\begin{array}{l}\text { Insulin sensitivity factor of } 2 \mathrm{mmol} / \text { unit implies that } 1 \text { unit } \\
\text { of insulin lowers the blood glucose by } 2 \mathrm{mmol} / \mathrm{L}\end{array}$ \\
\hline Insulin action time & $\begin{array}{l}\text { Time entered into the bolus calculator that insulin is } \\
\text { estimated to be active in the body (measured in hours) }\end{array}$ & Generally programmed anywhere between 3 and 6 hours \\
\hline Reservoir & Cartridge of insulin housed in the pump & \\
\hline Cannula & $\begin{array}{l}\text { Soft flexible tube or steel needle inserted into subcutaneous } \\
\text { tissue through which insulin is delivered into the body }\end{array}$ & \\
\hline Tubing or delivery line & Plastic tubing that connects cannula to reservoir & \\
\hline Infusion set & Includes cannula and tubing & \\
\hline $\begin{array}{l}\text { Continuous glucose } \\
\text { monitor }\end{array}$ & $\begin{array}{l}\text { Subcutaneous glucose sensor that measures glucose levels } \\
\text { in the subcutaneous fluid every few minutes. These may be } \\
\text { integrated with a pump }\end{array}$ & \\
\hline
\end{tabular}




\section{SELF-TEST}

\section{QUESTIONS}

True or false?

1. Patients using

an insulin pump

should change their

subcutaneous cannula

every three weeks.

2. A patient's daily insulin requirements are usually less when the drug is delivered by an insulin pump rather than injection.

Answers on page 205
This information is most efficiently obtained by uploading data from the insulin pump and incorporating a copy of the report in the patient's record. The data can be uploaded using web-based software to which the patient should have access, or directly from the screen on the pump.

A patient using an insulin pump may consult their GP while unwell and knowledge of the principles of sick-day management is important. As with the patient on multiple daily injections, those with type 1 diabetes should monitor their glucose and ketones closely. A temporary modification of usual basal insulin delivery (also known as a temporary basal rate) may be required on sick days. An increment in insulin delivery is usually required to deal with a rise in stress hormones increasing insulin resistance, but sometimes a reduction may be required if there is an associated diminished oral intake.

Sometimes an unexplained high glucose may be observed which is not explained by a pump malfunction. It is essential that blood ketones are checked. If moderately elevated ( $>0.4 \mathrm{mmol} / \mathrm{L}$ ), a corrective dose of rapid-acting insulin should be administered by injection as soon as possible. The insulin delivery line should then be changed and insulin delivery by the pump recommenced. If the ketones are critically elevated $(>1.6 \mathrm{mmol} / \mathrm{L})$ or the patient is nauseated and vomiting, they should be sent to an emergency department. Otherwise, the patient should check glucose and ketones hourly and further management should be determined in conjunction with a diabetes specialist team.

Occasionally there is a technical problem and the pump manufacturer's helpline should be contacted for assistance. When it is not safe for the patient to use the pump, and ketosis is not present, the patient will need to revert to multiple daily insulin injections until a replacement pump is available. The dosing for the multiple daily injections in these circumstances should be determined with the specialist team. If specialist advice is not available, the dose of insulin to be injected may need to be provided by the GP, as a temporary measure. As a rule, the average total basal insulin can be injected as long-acting insulin either once daily or preferably divided into two doses. Mealtime rapid-acting insulin doses can be estimated by using the patient's insulin-to-carbohydrate ratio and, if their glucose concentrations are high, by using their insulin sensitivity factor. However, insulin requirements for pump therapy are usually $20-25 \%$ less than with multiple daily injections and therefore this approach may underestimate insulin requirements, but it should be sufficient to avoid ketosis.

\section{Travelling with an insulin pump}

While travelling, an accompanying letter on practice letterhead should be provided stating the medical history of the patient, including the diagnosis of type 1 diabetes, and that management is via an insulin pump. A list of current drugs, with generic names and doses, should be included. The letter should stress that it is important that the patient's drugs accompany them, and that the insulin pump should not be disconnected. In particular, the pump should not be put through airport X-ray machines.

Prescriptions should be obtained for insulin and other drugs before travel. In case of pump failure both rapidacting and long-acting insulin should be obtained along with injecting devices and needles. Sufficient equipment for glucose testing should be packed in the hand luggage. In addition to drugs and glucosetesting equipment adequate quantities of consumables (batteries, insulin reservoirs, insulin delivery sets and sensors for continuous glucose monitoring) should be packed. As a rule, pack twice as many sets as potentially required for the planned period away from home. An adequate supply of consumables should be included in the hand luggage while flying.

\section{Conclusion}

Advances in technology have enabled insulin pumps to have a greater role in the management of type 1 diabetes. Successful management requires a team approach. $<$

Barbora Paldus has received research support and honoraria from Medtronic, Novo-Nordisk and Sanofi. Melissa Lee has received research support and honoraria from Medtronic. David O'Neal has received research support and is on advisory boards for Medtronic, Novo-Nordisk and Sanofi. He has received honoraria from Medtronic, AMSL, Novo-Nordisk and Sanofi.

\section{REFERENCES}

1. Pickup J, Mattock M, Kerry S. Glycaemic control with continuous subcutaneous insulin infusion compared with intensive insulin injections in patients with type 1 diabetes: meta-analysis of randomised controlled trials. BMJ 2002;324:705. https://doi.org/10.1136/bmj.324.7339.705

2. Australian Institute of Health and Welfare. Insulin pump use in Australia. Canberra: AlHW; 2012. www.aihw.gov.au/ reports/heart-stroke-vascular-disease/insulin-pump-use-inaustralia/contents/table-of-contents [cited 2018 Nov 1]

3. Best practice guide: Continuous subcutaneous insulin infusion (CSII). A clinical guide for adult diabetes services. ABCD Diabetes Technology Network UK; 2018. https://abcd.care/dtn-uk-best-practice-guides [cited 2018 Nov 1] 